\title{
A novel inhibitory anti-invasive MAb isolated using phenotypic screening highlights AnxA6 as a functionally relevant target protein in pancreatic cancer
}

Dermot O'Sullivan ${ }^{1}$, Paul Dowling ${ }^{2}$, Helena Joyce ${ }^{1}$, Edel McAuley ${ }^{1}$, Andrew McCann ${ }^{1}$, Michael Henry ${ }^{1}$, Brianan McGovern ${ }^{3}$, Paul Barham ${ }^{4}$, Fergal C Kelleher ${ }^{5}$, Jean Murphy ${ }^{3}$, Susan Kennedy ${ }^{3}$, Niall Swan ${ }^{3}$, Michael Moriarty ${ }^{1}$, Martin Clynes ${ }^{1,6}$ and Annemarie Larkin ${ }^{*, 1,6}$

${ }^{1}$ National Institute for Cellular Biotechnology, Dublin City University, Glasnevin, Dublin 9, Ireland; ${ }^{2}$ Department of Biology, National University of Ireland - Maynooth, Co. Kildare, Ireland; ${ }^{3}$ Department of Histopathology, St. Vincents' University Hospital, Elm Park, Dublin 4, Ireland; ${ }^{4}$ School of Biotechnology, Dublin City University, Glasnevin, Dublin 9, Ireland and ${ }^{5}$ Department of Medical Oncology, St. Vincents' University Hospital, Elm Park, Dublin 4, Ireland

Background: Discovery and validation of new antibody tractable targets is critical for the development of new antibody therapeutics to address unmet needs in oncology.

Methods: A highly invasive clonal variant of the MDA-MB-435S cell line was used to generate monoclonal antibodies (MAbs), which were screened for anti-invasive activity against aggressive cancer cells in vitro. The molecular target of selected inhibitory MAb 9E1 was identified using immunoprecipitation/liquid chromatography-tandem mass spectrometry. The potential anti-tumour effects of MAb 9E1 were investigated in vitro together with immunohistochemical analysis of the 9E1 target antigen in normal and cancer tissues.

Results: MAb 9E1 significantly decreases invasion in pancreatic, lung squamous and breast cancer cells and silencing of its target antigen, which was revealed as AnxA6, leads to markedly reduced invasive capacity of pancreatic and lung squamous cancer in vitro. IHC using MAb 9E1 revealed that AnXA6 exhibits a high prevalence of membrane immunoreactivity across aggressive tumour types with restricted expression observed in the majority of normal tissues. In pancreatic ductal adenocarcinoma, high AnxA6 IHC score correlated with the presence of tumour budding at the invasive front of tumours $(P=0.082)$, the presence of perineural invasion $(P=<0.0001)$ and showed a weak correlation with reduced survival $(P=0.2242)$.

Conclusions: This study highlights the use of phenotypic hybridoma screening as an effective strategy to select a novel functionblocking MAb, 9E1 with anti-cancer activity in vitro. Moreover, through characterisation of the 9E1 target antigen, AnxA6, our findings support further investigation of AnxA6 as a potential candidate target for antibody-mediated inhibition of pancreatic cancer.

New antibody-tractable molecular targets are urgently required for the development of effective bio-therapeutics for aggressive cancers with urgent unmet needs. Cell surface proteins represent highly attractive targets due to their accessibility; exploitation of candidate targets exhibiting high differential cancer-specific expression holds the potential to develop targeted antibodies such as antibody drug

*Correspondence: Dr A Larkin; E-mail: annemarie.larkin@dcu.ie

${ }^{6}$ These authors contributed equally to this work.

Received 9 June 2017; revised 17 July 2017; accepted 7 August 2017; published online 7 September 2017

(C) 2017 Cancer Research UK. All rights reserved 0007-0920/17 
conjugates (ADCs) which are capable of selective drug delivery to cancer cells without adversely affecting patients. Antibody drug conjugates and dual targeting strategies using bi-specific antibodies can improve clinical efficacy and overcome some of the limitations of first-generation monoclonal antibody (MAb)-based therapeutics.

Target agnostic approaches to identify functionally relevant targets are gaining momentum for identifying novel candidate drug targets, ultimately increasing the diversity of targets that will be tested clinically. High through-put target-based screening and omics approaches will not inform whether an antibody targeting approach will have a direct impact on tumour cells. Phenotypic hybridoma screening approach identifies candidate targets based on the desired cancer functional phenotype, thus having the potential to identify candidate proteins associated with a disease relevant biological response and may also lead to delineation of other disease targets/pathways. Functional screens have identified antibodies that can induce apoptosis, inhibit cell proliferation or internalise (reviewed by Gonzalez-Munoz et al, 2016). Such candidates are considered to have a higher likelihood of success in more advanced stages of drug development. We have previously demonstrated that a phenotypic MAb screening approach followed by mass-spectrometry identification of immunoprecipitated putative target antigens is an effective approach to identify candidate protein targets functionally involved in cancer invasion in vitro (O'Sullivan et al, 2014). Metastatic cancer is very challenging to treat due to its systemic nature and resistance to therapies. Thus, there is a continuing and urgent need to identify exploitable targets directly involved in the invasive and metastatic phenotype, which could have the potential to lead to the development of therapeutic strategies to prevent or delay metastasis.

Herein we report the generation and isolation of a novel function blocking MAb, designated 9E1, which was selected based on its ability to significantly suppress the invasive phenotype in vitro. This current study underscores the importance of such a functional target discovery strategy through the successful mass spectrometry identification of the functionally relevant molecular target of MAb 9E1 and its characterisation as a potential anticancer candidate protein target in a number of aggressive cancers.

\section{MATERIALS AND METHODS}

Cell lines. The MDA-MB-231, MDA-MB-468, MDA-MB-157, BT-20, BT474, HS78T, MCF-7, SKBR-3, MDA-MB-361, T-47D, NCI-H1299 and Lox-IVI cell lines were obtained from the American Tissue Culture Collection (ATCC, Rockville, MD, USA). The MiaPaCa-2 clone 3 pancreatic ductal adenocarcinoma (PDAC) cell line and the DLKPA and DLKP-M squamous lung carcinoma cell line were established in our laboratory (Clynes et al, 1992; McBride et al, 1998; Walsh et al, 2008).

Hybridoma generation. The immunogen chosen for $\mathrm{MAb}$ generation was MDA-MB-435-SF10p4p, an invasive clonal variant of the breast cell line, MDA-MB-435-S established in our laboratory (Glynn et al, 2004). Prior to immunisations, a nonenzymatic cell dissociation buffer was used to remove cells from flasks $\left(2 \times 10^{6}\right.$ cells $(50 \mu \mathrm{l}$ volume $\left.)\right)$ following which cells were washed and re-suspended in PBS plus an equal volume of lipopeptide adjuvant, Pam3Cys-SKKKK (EMC microcollections, Tubingen, Germany; L2000). Each mouse received a primary immunisation, followed by four booster immunisations administered at 3 weekly intervals. Three days prior to cell fusion mice received one final immunisation. Immunisations were carried out at the Royal College of Surgeons (RCSI) Biomedical Research Facility, Beaumont Hospital, Dublin 9, Ireland; all immunisation protocols were licensed by the Department of Health and Children, Ireland.

Screening of hybridomas. Following fusion hybridomas were screened directly for their ability to inhibit invasion in MDAMB435-SF cells and for their ability to show membrane immunoreactivity on un-fixed, non-permeabilised cells. Briefly, hybridomas were allowed to form large colonies and grow for at least 10 days undisturbed. All resultant hybridomas were screened initially, using a 96-well transwell Boyden chamber invasion assay and a standard 24-well assay for subsequent screenings. Cells were harvested at a concentration of $2.5 \times 10^{4} \mathrm{cells} \mathrm{ml}^{-1}$ in media (96well) or $1 \times 10^{6}$ cells ml $^{-1}$ (24-well), and $50 \mu \mathrm{l}$ (96-well) or $100 \mu \mathrm{l}$ ( 24 well) was added to each insert, along with an equal volume of antibody or control hybridoma medium (no antibody).

Immunofluorescence studies. Immunofluoresence staining was carried out as described previously with cells incubated overnight with supernatants and a fluorescein isothicyanate-linked (FITC) rabbit anti-mouse IgG secondary antibody (Dako, F0261 (O’Sullivan et al, 2014). Slides were viewed using a Nikon phase contrast microscope fitted with an FITC filter.

Invasion assays. For all 96-well invasion assays and preliminary 24well assays stained inserts were designated as having decreased levels of invasion or no effect on invasion relative to control hybridoma medium inserts (no hybridoma supernatant, representing 100\% invasion) (three independent evaluators). For later screenings and characterisation of MAb 9E1, the total number of invading cells was determined by counting the number of cells per field in 10 random fields at $\times 200$ magnification. The average number of cells per field was then multiplied by a factor of 140 as described previously (Walsh et al, 2008). (Note: due to inconsistency of invasion counts obtained with both MDA-MB-435-SF and MDA-MB-435-SF Taxol 10p4P cells, the extensively characterised cell lines, MiaPaCa-2 clone 3, DLKP-M and MBA-MB-231 was chosen to carry out all further $\mathrm{MAb}$ characterisation studies).

Motility assays. Motility assays were carried out in an identical manner to 24-well invasion assays, as described above, with the exception that the inserts were not coated in Matrigel.

Proliferation assays. Briefly, cells were harvested at $2 \times 10^{4}$ cells $\mathrm{ml}^{-1}$ in media and incubated at $37^{\circ} \mathrm{C}, 5 \% \mathrm{CO}_{2}$ overnight. Following this, $100 \mu \mathrm{l}$ of antibody was added to each well (control wells, $100 \mu$ l hybridoma media added) and incubated at $37^{\circ} \mathrm{C}, 5 \%$ $\mathrm{CO}_{2}$ for 6-7 days, until the control wells had reached $80-90 \%$ confluency. Assessment of cell survival in the presence of the antibodies relative to control (no MAb - hybridoma control medium only) was determined by the acid phosphatase assay.

Zymography. Zymography was carried out as described previously (O'Sullivan et al, 2014) with DLKP-M cells treated with MAb 9E1 $\left(50 \mu \mathrm{g} \mathrm{ml}^{-1}\right)$ or control cells (no MAb) for $24 \mathrm{~h}$ and concentrated conditioned medium analysed. Zones of gelatinolytic activity were detected as clear bands against a blue background.

Immunoprecipitation. Direct immunoprecipitation studies were performed with MAb 9E1 and control mouse IgG (Sigma, I5381) using protein L (P3351, Sigma, UK)/protein G agarose (Pierce, 22852). Purification, dialysis and concentration of MAb 9E1, preclearing of lysates, immunoprecipitation of the antibody-antigen complex, washing, elution and SDS-PAGE of 9E1 and mouse IgG immunoprecipitates were carried out as previously described (Larkin et al, 2005; O'Sullivan et al, 2014). Following gel electrophoresis, gels were stained with Brilliant Blue Colloidal Coomassie to visualise precipitated proteins. For validation of mass spectrometry identifications, gels were probed with relevant antibodies for western blotting (WB) analysis. 
Protein identification using liquid chromatography-tandem mass spectrometry. Liquid chromatography-tandem mass spectrometry was performed on an Ultimate 3000 nanoLC system (Dionex), interfaced to an LTQ Orbitrap XL (Thermo Fisher Scientific). Preparation of samples was based on Shevchenko et al (2006). Briefly, protein bands were excised from the Coomassiestained gel and destained. Samples were then dehydrated, digested and peptides were extracted with acetonitrile $/ 0.1 \%$ formic acid. A $5 \mu \mathrm{l}$ sample was loaded onto a trapping column, and the peptides were eluted into a C18 PepMAP100 nanocolumn. Peptides were then separated using mobile phase gradient. Liquid chromatography-tandem mass spectrometry data were acquired in datadependent acquisition mode controlled by Xcalibur 2.0.7 software (Thermo Fisher Scientific).

Bioinformatic profiling of identified proteins. Database searches were performed using TurboSEQUEST software (Bioworks Browser version 3.3.1) (Thermo Fisher Scientific) using the human subset from the SWISS-PROT database. The following filters were applied: for charge state $1, \mathrm{XCorr}>1.5$; for charge state 2, XCorr $>2.5$; for charge state 3 , XCorr $>3.5$.

RNA interference-mediated knockdown of AnxA6. Two predesigned siRNAs (Ambion) were chosen that target AnxA6 (Ambion, S1397 S1395), and transiently transfected into cells. Each set of siRNA transfections carried out was accompanied with a control (non-transfected) and a scrambled (SCR) transfection (Ambion, 4390843). Solutions of siRNA at a final concentration of $30 \mathrm{~nm}$ were prepared in OptiMEM (Invitrogen). siRNA experiments were set up using $2 \mu \mathrm{l} \mathrm{NeoFx} \mathrm{(Ambion,} \mathrm{AM4511)} \mathrm{to}$ transfect $30 \mathrm{Nm}$ siRNA at a cell density of $3 \times 10^{5}$ per $\mathrm{ml}$ in a sixwell plate. Transfection medium was removed after $24 \mathrm{~h}$ and replaced with fresh growth medium. A Kinesin-specific siRNA (Ambion) was used a positive control in all knockdown experiments to assess the efficiency of transfections. The transfected cells were collected at 72 and $96 \mathrm{~h}$ for immunoblotting and assayed for changes in invasion capacity at $24 \mathrm{~h}$ as described above.

Immunoblotting. SDS-PAGE, WB and ECL development was carried out following extraction of whole cell lysates from relevant cell lines and BCA protein concentration determination as previously described (O'Sullivan et al, 2014). Enriched membrane fractions of cell lines were isolated using the Calbiochem Native membrane protein kit (444810), according to the manufacturer's guidelines. The following antibodies were used: MAb 9E1 supernatants, AnxA6 (Abcam, ab5221, dilution 1:5000), Prohibitin (Calbiochem, CP34, dilution, 1:800); 14-3-3 epsilon (Novus Biologicals, NBPI 32695, 1:1000); beta-actin (Sigma, AS441, dilution 1:10000); $\alpha$-Tubulin (Sigma, T5168, dilution 1:1000), phospho and total ERK, MEK and p70SK6 (Cell Signalling, $4370,4695,2338,9122,9204$ and 9202, dilution $1: 1000$ or $1: 2000(2338))$.

Immunohistochemistry. Immunohistochemical staining of full face $5 \mu \mathrm{m}$ sections and TMAs (lung cancer, LC72ai, US Biomax) was performed using the Dako Autostainer Plus (Dako) according to the manufacturer's guidelines. Prior to staining, slides were subjected to an antigen retrieval step consisting of a 20-min incubation in Target Retrieval, ph 6, Dako S1699 for Ki-67 and a 15-min incubation in Target Retrival pH 9, Dako S2375 for 9E1. Primary antibodies: MAb 9E1, (concentration: $10 \mu \mathrm{g} \mathrm{ml}^{-1}$ ) and MAb Ki67 (Clone MIB1, M7240, Dako, dilution 1:30) and negative controls (primary antibody omitted, replaced by TBS) were processed at the same time. Archival melanoma, breast, PDAC, colon and renal tumours and control normal tissues were obtained courtesy of the Departments of Pathology, St Vincents' University Hospital (SVUH) Dublin and the Royal Victoria Eye and Ear Hospital (RVEEH) Dublin. Ethical approval for IHC analyses of tissues was obtained from the SVUH and RVEEH ethics committees. Clinicopathological details were obtained from patient records.

Immunohistochemistry scoring. MAb 9E1 immunoreactivity to assess AnxA6 expression across normal and cancer tissues was scored as absent, weak (faint cytoplasmic or nuclear staining of scattered cells), moderate (intermediate or heterogenous membrane staining in tumour cells/observed across tissue) or strong (intense membrane staining in the vast majority of tumour cells/ observed across tissue). A semi-quantitative scoring system (based on the staining intensity and the percentage of tumour cells stained) was used to evaluate AnxA6 expression in the PDAC cohort. Staining intensity was assigned as follows: $(1+$, weak; $2+$, moderate; $3+$, intense; $4+$, very intense). The proportion of tumour cells stained was scored as follows: $(1=<5 \% ; 2=5-49 \%$; $3=50-69 \% ; 4=>70 \%$ of tumour cells). AnxA6 expression was assigned by combining the staining intensity scores and the proportion of tumour cells stained. The minimum score when summed (intensity and percentage) was 1 and the maximum score was 8. Patients were stratified into two clinical score categories high AnxA6 expressing (overall score 5-8) or low AnxA6 expressing (overall score 1-4). Scoring was carried out by two independent evaluators (AML, EMcA) blinded to specimen and clinical detail and was also reviewed by a Consultant pathologist (NS).

Statistical analysis. All data are presented as mean \pm standard deviation (s.d.). Analysis of the difference of comparisons, scrambled control siRNA versus siRNA treated mean invasion and motility counts, adherence absorbance, and percentage survival calculated, were performed using the student's $t$-test (two-tailed, unpaired with equal variance). A $P$-value of $\leqslant 0.05^{\star}$, $\leqslant 0.01^{\star *}, \leqslant 0.005^{\star * \star}$ was deemed statistically significant. Statistical analysis was performed using Microsoft Excel and Prisim Graphpad Software. All experiments were repeated a minimum of three times; with the exception of initial hybridoma invasion screenings (96-well and 24-well), preliminary immunofluoresence studies and IHC analyses.

\section{RESULTS}

Isolation of anti-invasive hybridomas using functional screening. Five supernatants were chosen on the basis of a decrease in invasion in MDA-MB-435 SF cells observed compared to hybridoma control medium. Fifteen supernatants were selected on the basis of their reactivity with MDA-MB-435 SF cells using live cell immunofluorescence. Following expansion into 6-well plates, and re-screening (invasion assays and immunofluorescence), hybridomas 4E5, 9E1, 102C, 1D2 and 104D were chosen for further characterisation.

Selection of inhibitory MAb 9E1. MAbs 4E5 and 9E1 were cloned by limiting dilution, the clone designated 24(6) arising from the parental hybridoma, 9E1 was selected for further study as it showed the most consistent anti-invasive effects and exhibited 'punctate'-like membrane associated staining on non-permeabilised cells (Figure 1) (note: MAb 9E1 clone 24(6) is referred to, throughout this manuscript as MAb 9E1, its parental name). The isotype of antibody 9E1 was revealed to be IgG1 (data not shown).

Quantification of MAb 9E1. Following isotype analysis which revealed the isotype of antibody $9 \mathrm{E} 1$ as an $\mathrm{IgG1}$, a panel of MAb 9E1 supernatants were then quantified using the Mouse IgG ELISA Quantitation Set from Bethyl laboratories (USA) (Cat. No. E90131). These ranged from $50 \mu \mathrm{g} \mathrm{ml}^{-1}$ to $103 \mu \mathrm{g} \mathrm{ml}^{-1} \mathrm{IgG}$. An excellent linear standard curve was obtained with MAb 9E1 
(concentration range $0-160 \mathrm{ng} \mathrm{ml}^{-1}$ ) (data not shown). Dose response studies on inhibitory effects of MAb 9E1 in MiaPaCa2clone 3 and DLKP-M cells demonstrated a dose response effect in both cell lines over a range of concentrations (10-100 $50 \mu \mathrm{g} \mathrm{ml}^{-1}$ ) (Figure $1 \mathrm{C}$ and Supplementary Figure $1 \mathrm{~B}(\mathrm{i})) .50 \mu \mathrm{g} \mathrm{ml}^{-1}$ of antibody led to significant inhibition of invasion in both cell lines; thus this concentration of MAb was used for all of the following characterisation studies.

Immunofluorescence studies of MAb 9E1 in cancer cell lines. Immunofluorescence staining to probe MAb 9E1 binding specificity and cellular localisation of its target antigen revealed 'punctate'-like membrane immunoreactivity across cell lines, SKBR3, BT-474 (Figure 1D) DLKP-M, MiaPaCa-2 clone 3, MDA-MB-231 and MDA-MB-435 cell lines (data not shown). Taken together with WB reactivity observed in membrane enriched fractions, these results strongly suggested that the putative target antigen of selected inhibitory MAb 9E1 was a membrane candidate protein.

Confirmation of specificity against cancer cell lines. Whole cell lysates and membrane enriched fractions of breast cancer (Supplementary Figure 1), colon, lung and PDAC cell lines (data not shown) revealed that MAb 9E1 showed reactivity with an approximately $65 \mathrm{kDa}$ band across all cell lines (Supplementary Figure 1).

MAb 9E1 impedes the invasive potential of aggressive cancer types (breast, PDAC, squamous lung) in vitro. Further cancer cell models were investigated to ascertain if antibody 9E1 could also impede invasion in other aggressive cancers; the most pronounced effects were observed in MiaPaCa-2 clone 3, a highly invasive clonal population isolated from the parental MiaPaCa-2 PDAC cell line (mean inhibition level 79.6\%) (Figure 1C and Supplementary Figure 2) and DLKP-M squamous lung carcinoma
A

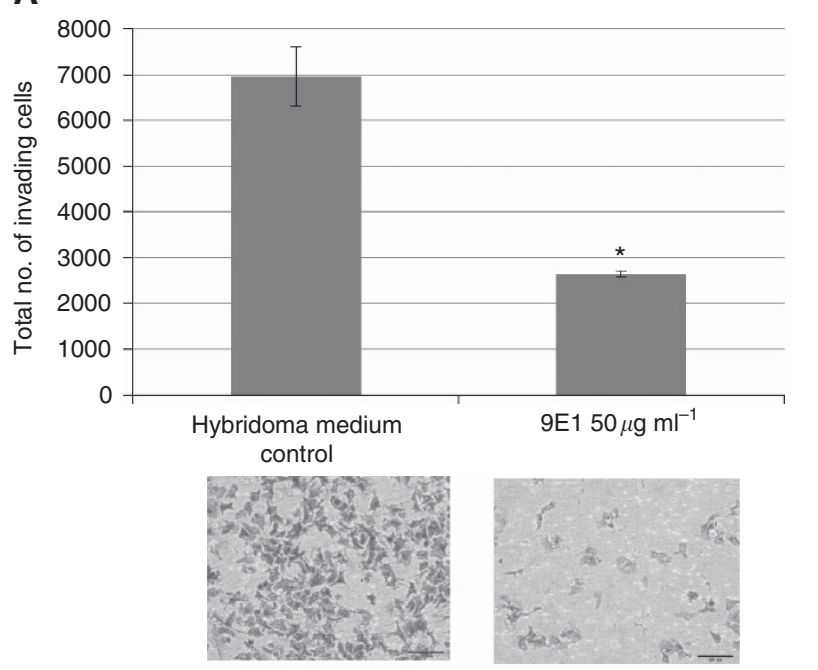

B

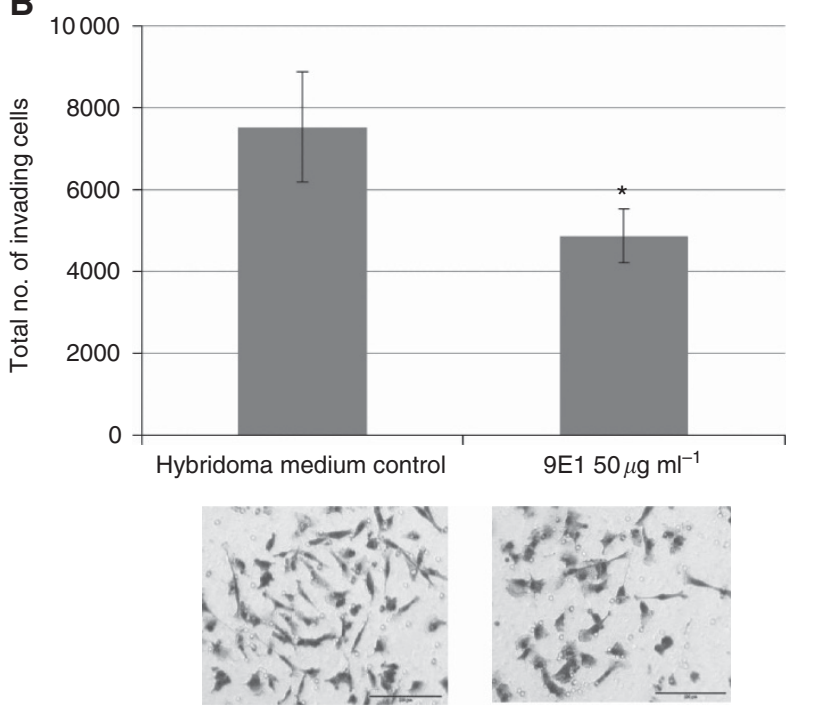

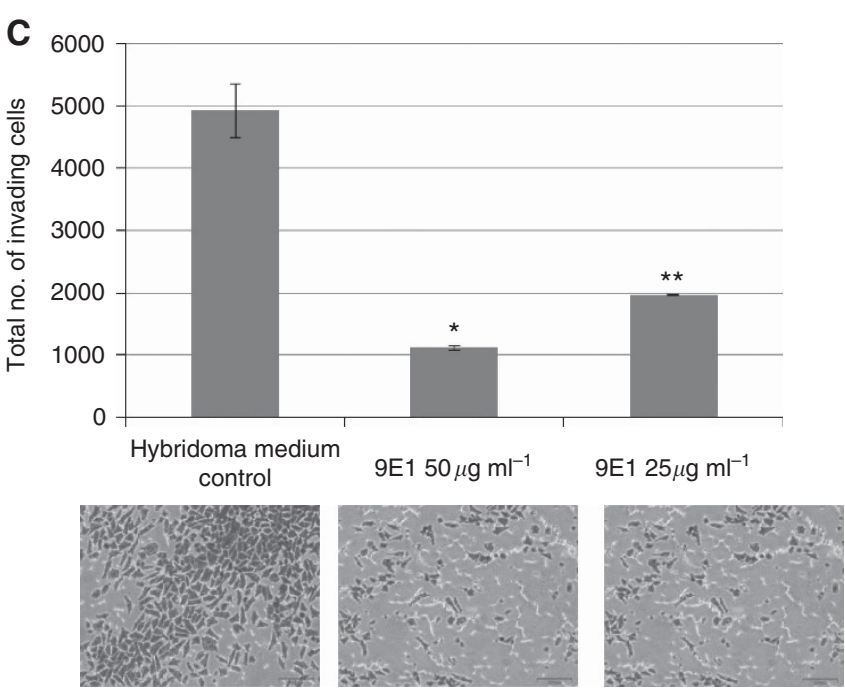

D

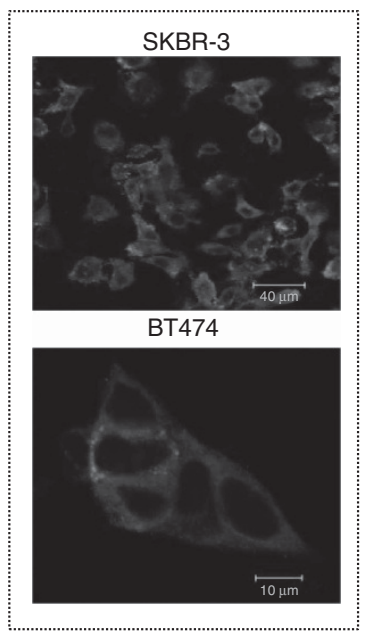

Figure 1. The invasive potential of DLPK-M (A), MDA-MB-231 (B) and MiaPaCa-2 clone 3 (C) cells is significantly reduced in the presence of $50 \mu \mathrm{g} \mathrm{ml}^{-1}$ of $9 \mathrm{E} 1 \mathrm{MAb}$, and to a lesser extent in the presence of $25 \mu \mathrm{g} \mathrm{ml}^{-1}$ of MAb indicating a dose response effect. Data plotted represent the mean \pm standard deviation from a representative experiment. Statistics: ${ }^{\star} P \leqslant 0.05,{ }^{\star} P \leqslant 0.01$, compared to hybridoma medium control (no MAb). Student's t-test (two-tailed with equal variance, unpaired). Representative photomicrographs of invading cells are also shown ( \pm Mab) (Original magnification, $\times 100$; scale bar $=200 \mu \mathrm{m}$ ). All experiments were performed a minimum of three times, and representative results are presented. (D) 'punctate'-like 9E1 immunoreactivity is observed in un-permeabilised SKBR3 and BT-474 cells. 
cells, a highly invasive clonal population isolated from the parental DLKP cell line (mean inhibition level of 76.2\%) (Figure 1A). The inhibitory effects of MAb 9E1 in the other cancer cell lines were not as marked but were significant: SKBR-3 (mean inhibition level of 55.6\%), MDA-MB-231 (mean inhibition level of 53.3\%) (Figure 1), LOX-IVI melanoma (mean inhibition level of 50\%), NCI-H1299 large cell lung (mean inhibition level of 34.5\%) (Supplementary Figure 3).

Identification of the MAb 9E1 target antigen. In order to identify the putative target antigen of MAb 9E1 direct immunoprecipitation followed by protein identification by mass-spectrometry was performed. We choose three cell lines with abundant 9E1 target expression: MiaPaCa-2 clone 3, NCI-H1299 and DLKPA. Bands were revealed at approx. $175,155,65,42$ and $30 \mathrm{kDa}$ in all cell lines (Figure 2A and B). Peptides from mass spectrometry identifications were searched using the SWISS-PROT database. Bands 1 and 2, while not apparent on gels with control mouse IgG immunoprecipitates, were identified as mouse IgG. No definitive identification could be obtained for band 4. A number of hits were obtained from the $30 \mathrm{kDa}$ reactive band (Supplementary Table 1); prohibitin, ADP/ATP translocases 1, 2 and 3, 14-3-3 epsilon and 40S Ribosomal Proteins,
3 and S4 isoforms, Y1, Y2 and X. Prohibitin and 14-3-3 epsilon proteins identifications were validated successfully with commercial antibodies (data not shown). As WB analyses across a number of cell lines had revealed only one reactive band at approximately $65 \mathrm{kDa}$, we speculated that this band may represent the 9E1 target antigen. This band was identified as AnxA6. As expected, when MiaPaCa 2 clone 3, H12929 and DLKPA immunoprecipitates were probed with 9E1, only the $65 \mathrm{kDa}$ band was identified. A representative MiaPaCa-2 immunoprecipitate probed with an AnxA6 specific antibody is shown in Figure 2C, identifying the MAb 9E1 target antigen at approximately $65 \mathrm{kDa}$, as AnxA6. Immunoblots were also run in parallel and probed with MAb 9E1 which identified also the $65 \mathrm{kDa}$ reactive band (data not shown). A marked reduction in expression of the AnxA6 and 9E1 reactive bands were observed in AnxA6 siRNA transfected cells probed with a commercial AnxA6 antibody and MAB 9E1, thus confirming AnxA6 as the 9E1 MAb target antigen (Figures 2D, 3A and B).

MAb 9E1 effects on MMP-9 activity in MDA-MB-231 TNBC cells. Zymographic analysis of conditioned medium from MDAMB-231 cells treated with antibody 9E1 revealed a reduction in MMP-9 compared to conditioned medium from cells treated with hybridoma medium only (control) (Supplementary Figure 1).
A

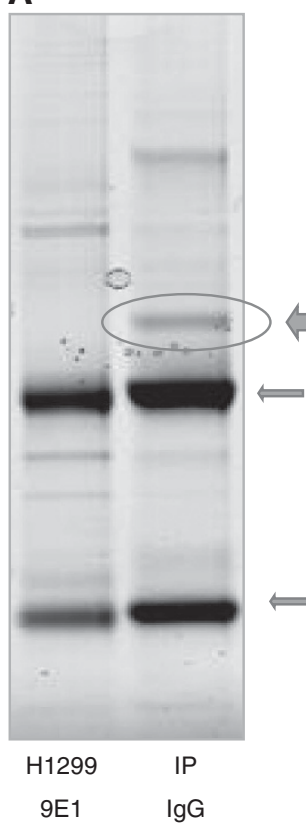

D $65 \mathrm{kDa} \square$
B

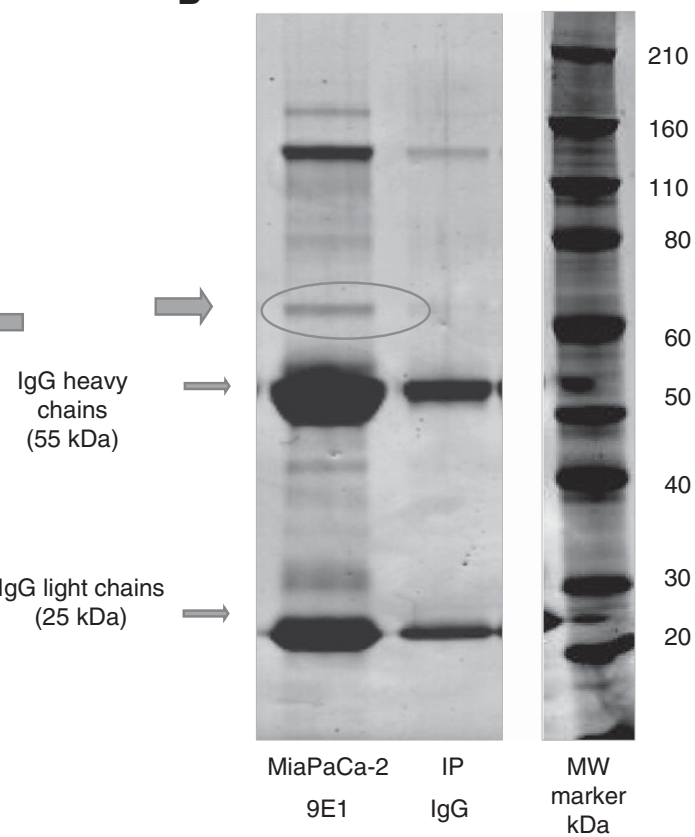

C

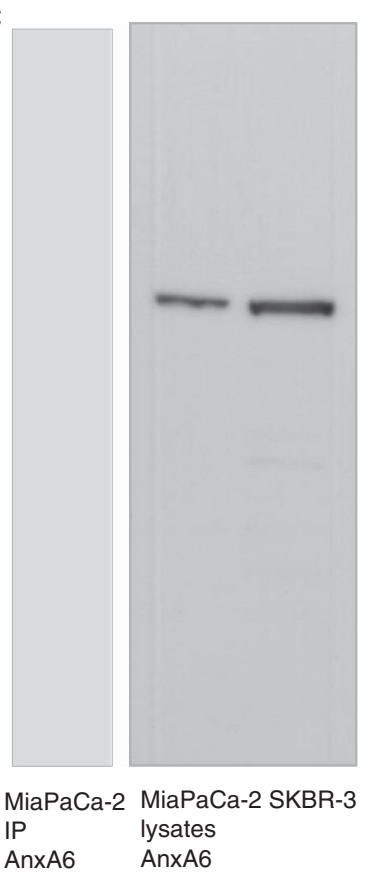

Control Scrambled siRNA $\alpha \quad$ siRNA $\beta$
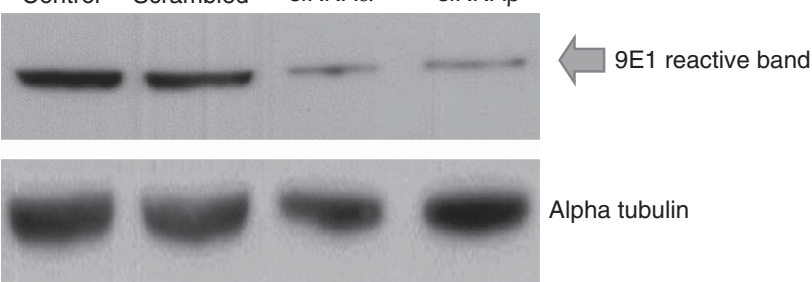

Figure 2. Identification of the 9E1 target antigen. Representative SDS-PAGE gels showing (A) control mouse IgG and NCl-H1299; (B) MiaPaCa-2 clone 3 cells and control mouse IgG directly immunoprecipitated with MAb 9E1. Five bands were observed, at approx. 170, 155, 65, 42 and $30 \mathrm{kDa}$, which were not present in the control mouse IgG immunoprecipitates (IP). Each band was excised and run through an LCMS/LTQ mass spectrometer for identification (Table 2). Experiments were carried on biological triplicate samples and on other cell lines (LOX-IVI, DLKPA) (results not shown). Validation of the MAb target antigen as AnxA6 (65 kDa) is shown in (C): MiaPaCa-2 clone 3 IP probed with an AnxA6 specific commercial antibody reveals a reactive band at $65 \mathrm{kDa}$. MiaPaCa-2 clone 3 and SKBR3 lysates also shown probed with AnxA6, (D) MiaPaCA-2 clone 3 cells transfected with AnxA6 siRNAs ( $a$ and $b$ ) probed with MAb 9E1 showing a marked reduction in expression of the 9E1 reactive band relative to siRNA scrambled control. (loading control, alpha tubulin) $(n=3)$. 
These results indicated that 9E1 may possibly be eliciting its antiinvasive effects through reduction of MMP-9 in MDA-MB-231 cells.

MAb 9E1 effects on the ERK-MEK signalling pathway in DLKPM cells. To further investigate the potential mechanism by which $\mathrm{MAb}$ 9E1 may be reducing invasion we sought to examine the effects of 9E1 antibody on the ERK-MEK/PK13S kinase signalling pathways. The DLKP-M cell line was chosen to carry out this study as MAb 9E1 treatment had significantly reduced invasion in a dose responsive manner in this cell line and previous work in our laboratory had implicated the ERK-MEK pathway in the invasive phenotype of these cells. Inhibitory MAb 9E1 treatment resulted in activation of ERK signalling (and MEK) (Supplementary Figure 4) with possible reduction in activation of P70S6 kinase suggesting that MAb 9E1 may be eliciting its anti-invasive effects, at least in part, through modulation of these two pathways. These effects were not observed upon treatment, carried out in parallel with $50 \mu \mathrm{g} \mathrm{ml}^{-1}$ of an irrelevant IgG MAb.

RNA interference mediated knockdown of the 9E1 target antigen, AnxA6 suppresses the invasive capacity of MiaPaCa-2 clone 3 and DLKP-M cells. Leading on from MAb 9E1's inhibitory effects on invasion, we then determined the effects of siRNA-mediated knockdown to establish if AnxA6 is functionally involved in cancer in vitro. Downregulation of AnxA6 significantly reduced the invasive capacity of MiaPaCa-2 clone 3 and DLKP-M cells (Figure 3). In agreement with MAb blocking experiments no significant effect on proliferation or motility in either cell line was observed following AnxA6 silencing, suggesting that the observed reductions in invasion were not just reflecting altered cell proliferation. Thus, AnxA6 is functionally involved in the invasive phenotype of PDAC and lung squamous cancer in vitro. AnxA6 transfected cells were also probed with MAb 9E1; a marked reduction in the $65 \mathrm{kda}$ MAb reactive band was observed in lysates transfected with siRNAs (Figure 2D ( $a$ and $b$ )).

Immunohistochemistry analysis of AnxA6 using MAb 9E1. AnxA6 (as indicated by MAb 9E1 moderate-strong immunoreactivity) shows a high prevalence of expression across colon, PDAC, breast cancer and lung cancer sub-types with restrictive expression across normal tissues (as indicated by very weak 9E1 immunoreactivity in $<10 \%$ of tissue) and highly proliferating cells and tissues (normal colon, skin and tonsil) (Table 1). Collectively these results suggested that AnxA6, the 9E1 target antigen was selective towards cancer tissues with minimal reactivity across normal and highly proliferating cells and tissues.

Correlation of AnxA6 expression with clinicopathological features in PDAC. Clinicopathological features of 57 patients diagnosed with PDAC between 2007 and 2013 are presented in Table 2. AnxA6 expression was observed in 57 of 57 PDAC tumours. Tumours were stratified as either highly AnxA6 expressing (IHC score $3+/ 4+$ ) or weakly AnxA6 expressing (IHC score $1+/ 2+$ ). A highly significant correlation was shown between high AnxA6 IHC score $(3+/ 4+)$ and the presence of perineural invasion (PNI) $(P=<0.0001)$. High AnxA6 score also correlated with tumours exhibiting tumour budding ( $v s$ no tumour budding) $(P=0.0827)$.

No significant association with sex, age, tumour size, histological grade, node status or presence of lymphovascular invasion was shown.

Kaplan-Meier survival analysis of AnxA6 with outcome in PDAC. Kaplan-Meier survival analysis carried out using GraphPad PRISM revealed a clear trend towards high AnxA6
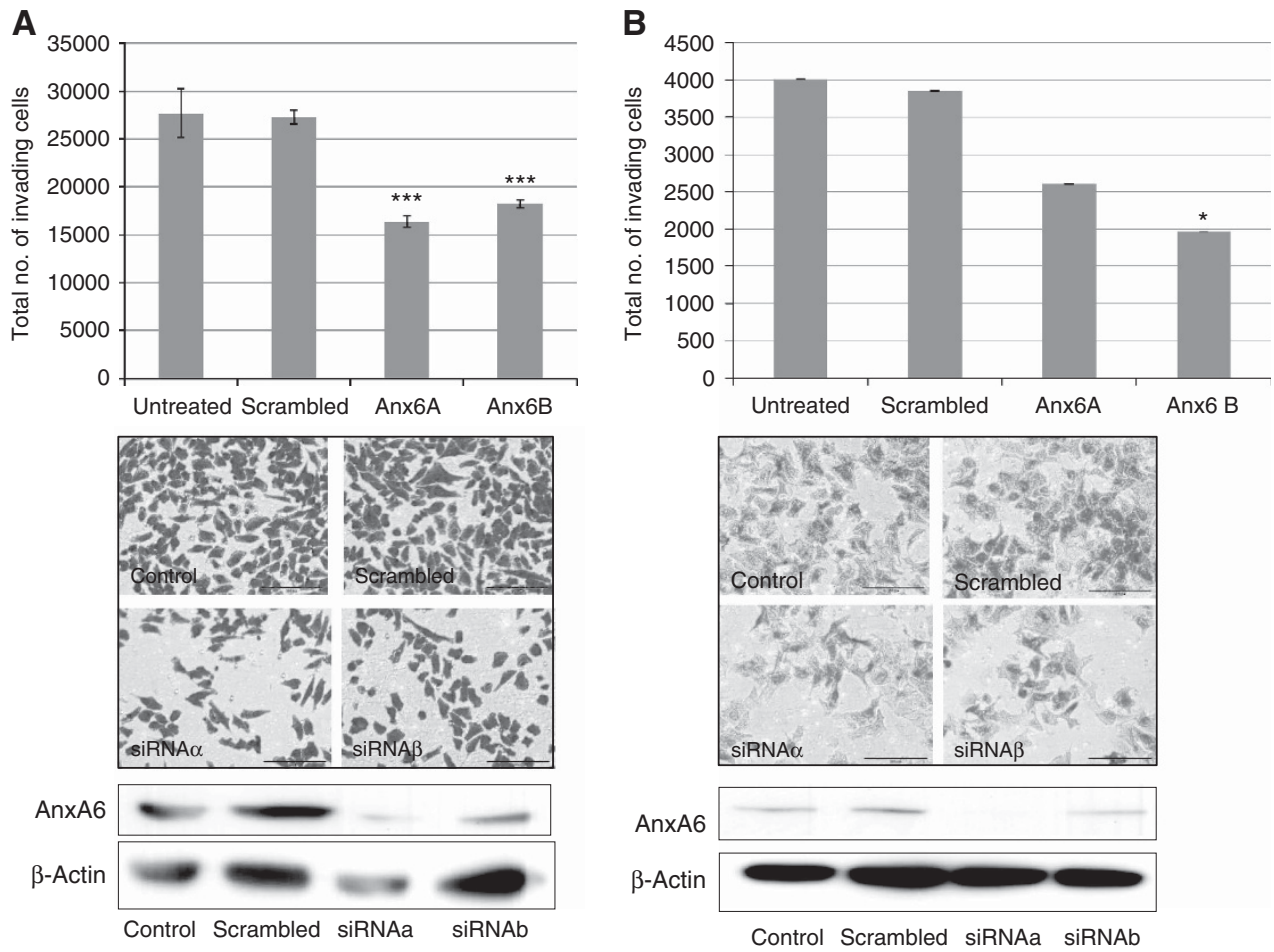

Figure 3. Knockdown of AnxA6 reduces invasive capacity of MiaPaCa-2 clone 3 and DLKP-M cells. (A) Representative histogram showing reduced invasive capacity of (A) MiaPaCa-2 clone 3 cells and (B) DLKP-M cells in a Boyden chamber assay following transfection with Anx-6 siRNAs, a and b. Data plotted represent the mean \pm standard deviation of duplicate transwell inserts from a representative experiment. Statistics: ${ }^{*} \leqslant 0.05$, $\star \star \star P \leqslant 0.005$, compared to scrambled siRNA control; Student's t-test (two-tailed with equal variance, unpaired). A representative immunoblot is shown indicating siRNA-mediated knockdown of AnxA6 in MiaPaCa-2 clone 3 and DLKP-M cells. Representative photomicrographs showing invasion status of MiaPaCa-2 clone 3 cells following transfection are also shown. Original magnification, $\times 10 ;$ scale bar $=200 \mu \mathrm{m})$. All experiments were performed a minimum of three times and representative results are presented. 
Table 1. IHC analysis of AnxA6 expression in normal tissues and cancer tissues using MAb 9E1

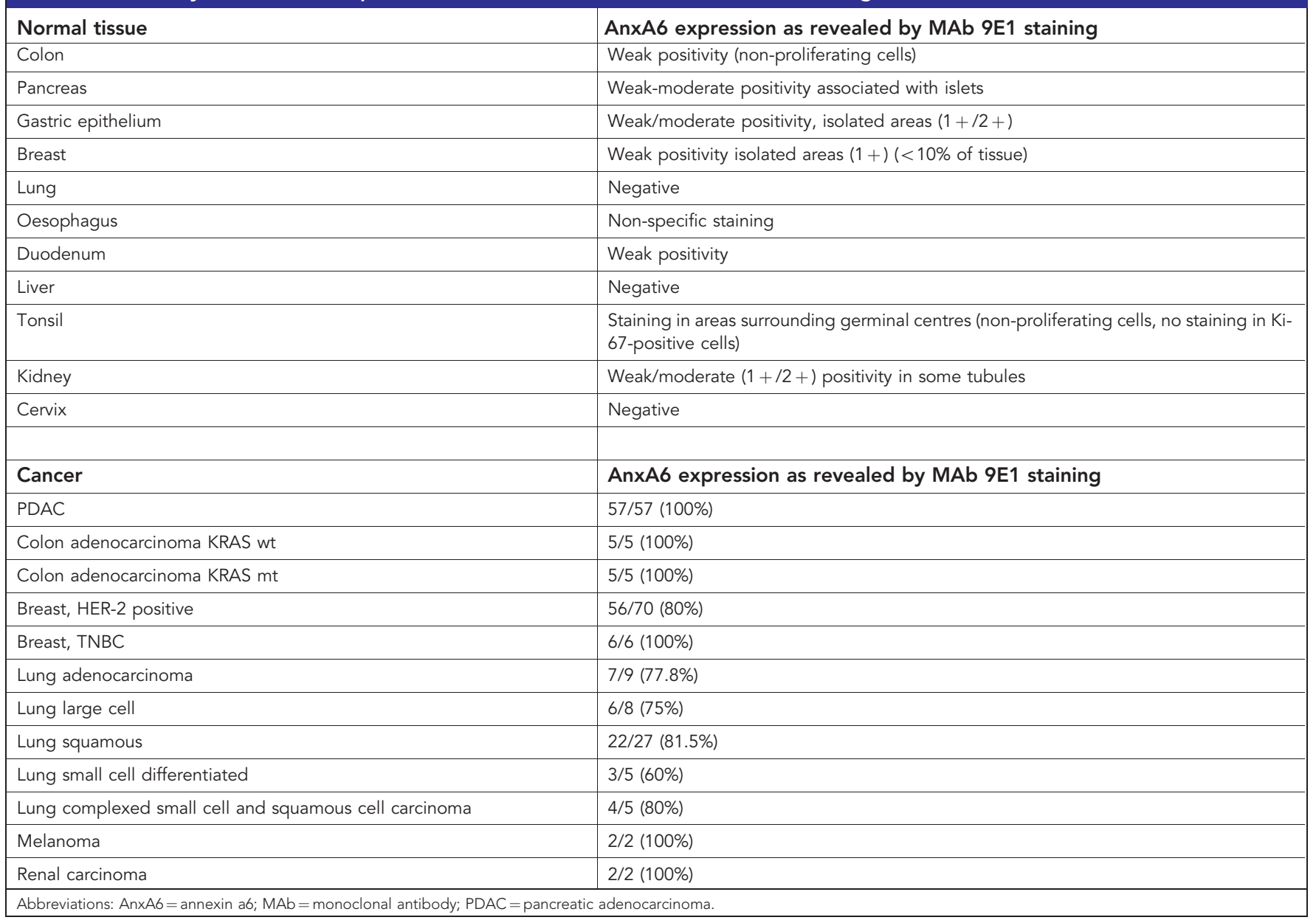

expression (IHC score $3+/ 4+$ ) being associated with a poorer outcome in the 57 PDAC cohort $(P=0.2242)$; however, this failed to reach significance. The study group, $n=57$, was relatively small; thus, significance may be achieved if expression is studied in a larger patient cohort. Median survival of patients whose tumours exhibited high AnxA6 $(3+/ 4+)$ expression was 632 days vs 998 days for patients whose tumours exhibited a lower AnxA6 IHC score $(1+/ 2+)$ (Figure 4$)$.

\section{DISCUSSION}

Phenotypic, target agnostic hybridoma screening is an attractive strategy for novel antibody target discovery, with demonstrated potential for clinical translation (Kinch et al, 2009; Dudley et al, 2014; Sandercock et al, 2015). Using such an approach candidate $\mathrm{MAb} 9 \mathrm{E} 1$ was selected due to its potent inhibitory anti-invasive effects across a number of aggressive cancer types (PDAC, lung, melanoma and breast) and based on its 'punctate'-like membrane associated immunoreactivity on non-permeabilised cells (Figure 1). MAb 9E1 reacted with a band at approximately $65 \mathrm{kDa}$ across cell lines. We thus speculated that this band represented the 9E1 target antigen, which we subsequently confirmed with direct immunoprecipitation studies. Liquid chromatography-tandem mass spectrometry identified the $65 \mathrm{kDa}$ band as AnxA6 (Figure 3), which we then validated by WB analysis. AnxA6 appears to have a dichotomous role in cancer, displaying tumour suppressor and tumour potentiating effects across various cancer types (Qi et al, 2015; Grewal et al, 2017). Here, siRNA-mediated knockdown of
AnxA6 significantly reduced the invasive capacity of both MiaPaCa-2 clone 3 and DLKP-M cells, and thus implicates AnxA6 in PDAC and lung squamous cancer invasion in vitro. It is noteworthy that probing of AnxA6 silenced cells demonstrated that the 9E1 reactive band was markedly reduced providing further evidence that AnxA6 was indeed the 9E1 molecular target. siRNA knockdown studies have previously implicated AnxA6 in tumour growth of gastric cancer cells and in breast cancer cell lines with low levels of AnxA6 expression (Sakwe et al, 2011; Koumangoye et al, 2013; Wang et al, 2013). Reduced expression of AnxA6 appears to inhibit motility and invasiveness of breast cancer cells, supporting a tumour suppressor role for AnxA6 in breast cancer. Perhaps AnxA6 is involved in proliferation in the context of its tumour suppressor capacity and when acting as a pro-oncogene, it is not directly contributing to proliferation, at least in the in vitro setting. Obviously it may be involved in tumour growth in vivo as cell signalling cross-talk between the tumour and the tumour environment may impact on its mechanism of action. However, our results clearly demonstrate that antibody-mediated targeted blocking of AnxA6 can suppress the invasive phenotype in vitro.

AnxA6 has been shown to interact with Ras-ERK-MEK and/or P13K pathways. siRNA-mediated suppression of AnxA6 was previously shown to lead to enhanced EGF-induced Ras and ERK activation in Hela cells (Grewal et al., 2005). Sakwe et al. showed that AnxA6-dependent breast cancer adhesion and motility were dependent on activation of the FAK-P13-AKT pathway. We postulated therefore that mechanistically, MAB 9E1's blocking activity may be modulated via these signalling pathways. As expected, we demonstrated that MAb 9E1 treatment leads to 
A

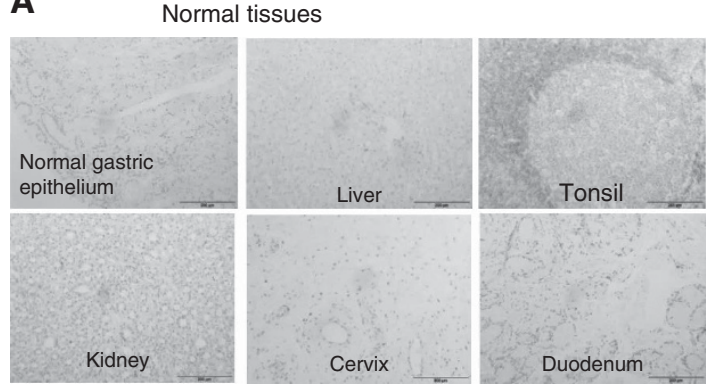

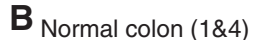

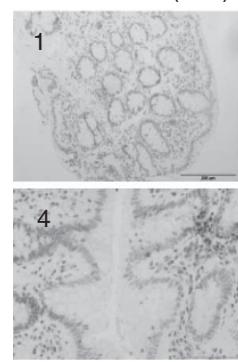

Colon adenocarcinoma $(2,3,5$ \& 6$)$
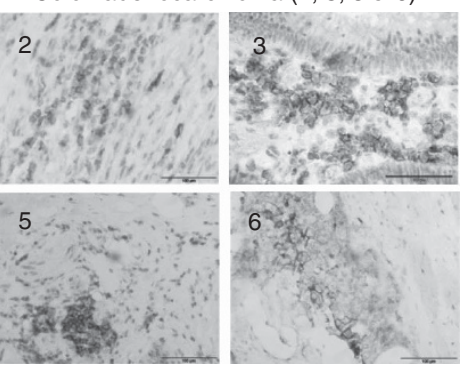

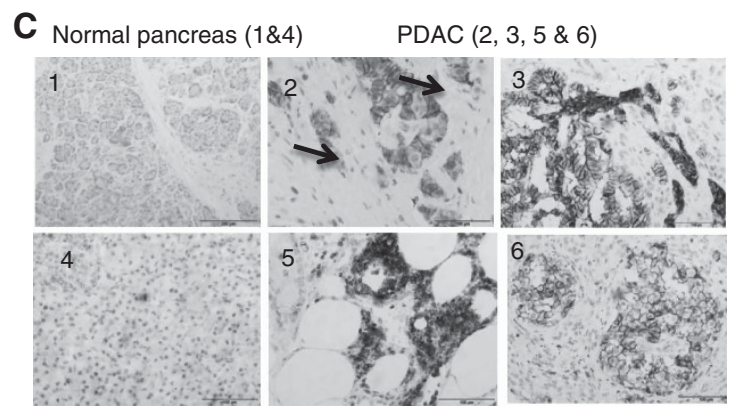

High AnaxA6 intensity (3+/4+) score vs low intensity $(1+/ 2+)$

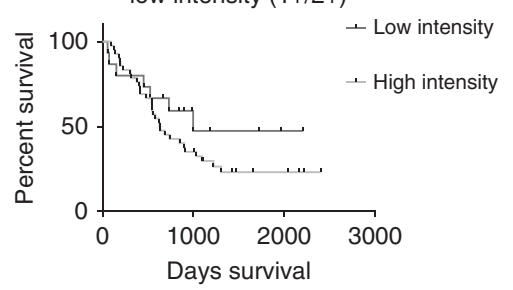

Figure 4. IHC analysis with MAb 9E1 demonstrates that AnxA6 shows limited expression in normal and highly proliferating tissues (A) with strong membrane AnxA6 expression observed in colon adenocarcinoma (B) and PDAC (C). High AnxA6 expression was significantly associated with the presence of PNI $(P=0.0001)$ and with tumours exhibiting tumour budding $(P=0.0082)$ in PDAC and showed a weak correlation with outcome $(P=0.2242)$ in this 57 PDAC cohort.

activation of phospho-ERK while at the same time leads to possible reduction in P70S6K activation. Interestingly immunoprecipitation/mass spectrometry did identify the scaffold protein, prohibitin (PHB) as an interacting protein of AnxA6 in this study. PHB forms a signalling complex with CRAF at the cell surface to regulate MEK-ERK activation in PDAC (Luan et al, 2014). We also demonstrated that MAb 9E1 treatment leads to reduction in MMP-9 activity in MDA-MB-231 cells. These results suggest that perhaps MAb 9E1 (following binding to AnxA6) is exerting its anti-invasive effects, in part, through modulation of the ERK signalling axis involving MMP-9. ERK signalling has been shown to modulate MMP-9 activity in MDA-MB-231 invasion (Moulik et al, 2014). Future work is planned to eluciadate 9E1's mechanism of action following binding to its target antigen, AnxA6. Using $\mathrm{MAb} 9 \mathrm{E} 1$ we demonstrated that AnxA6 is highly expressed in a number of aggressive cancers (Table 1) namely, PDAC, lung squamous, colon adenocarcinoma, TNBC and HER-2 positive breast cancers. A concern for any potential therapeutic antibody targeting approach is the potential for off-tumour toxicities due to expression of the candidate protein in normal tissues. Restricted AnxA6 expression as indicated by weak/negligible 9E1 immunoreactivity was observed in the vast majority of normal tissues representing vital organs studied (Table 1 and Figure 4). Membrane AnxA6 immunoreactivity was observed in islet cells in pancreas and in isolated cells in duodenum and gastric epithelium and in some kidney ducts; however, in all cases weak to moderate staining only was revealed and was not homogenously observed across tissues. In addition to differential and selective expression, minimal expression of the potential ADC molecular target in highly proliferating cells is essential. Our studies demonstrate that AnxA6 expression is not associated with highly proliferating, Ki-67 positive cells and would fulfil this particular criteria.

A potential molecular target should be abundantly and selectively expressed on the cell surface. Our preliminary experiments indicated that the 9E1 target antigen was observed in membrane enriched fractions across tumour cell lines and we observed 'punctate'-type 9E1 immunoreactivity, suggesting a potential association with vesicular structures, perhaps exosomes. Given the fact that MAb 9E1 markedly reduces the invasive capacity of tumour cells, it was likely thus that the putative molecular target of MAb 9E1 was likely to be a cell surface protein. Indeed, AnxA6 has been implicated as a multifunctional scaffold protein, recruiting signalling proteins, modulating cholesterol and membrane transport and influencing actin dynamics, all of which require AnxA6 to be localised at either the plasma membrane or endocytic compartments (Pons et al, 2001; Koese et al, 2013; Koumangoye et al, 2013). AnxA6 has also been observed in membrane protrusions of breast cancer cells. Interestingly a recent study by Leca $e t$ al. has identified a complex of AnxA6/LRP1/TSP1 in cancer-associated fibroblasts (CAFs) in PDAC and demonstrated that uptake of CAF-derived AnxA6 is directly linked to PDAC aggressiveness (Leca et al, 2016). In the context of investigating AnxA6 as a possible candidate for targeting using 
Table 2. IHC analysis of AnxA6 expression in PDAC $(N=57)$ using MAb 9E1 and correlation with clinicopathological features

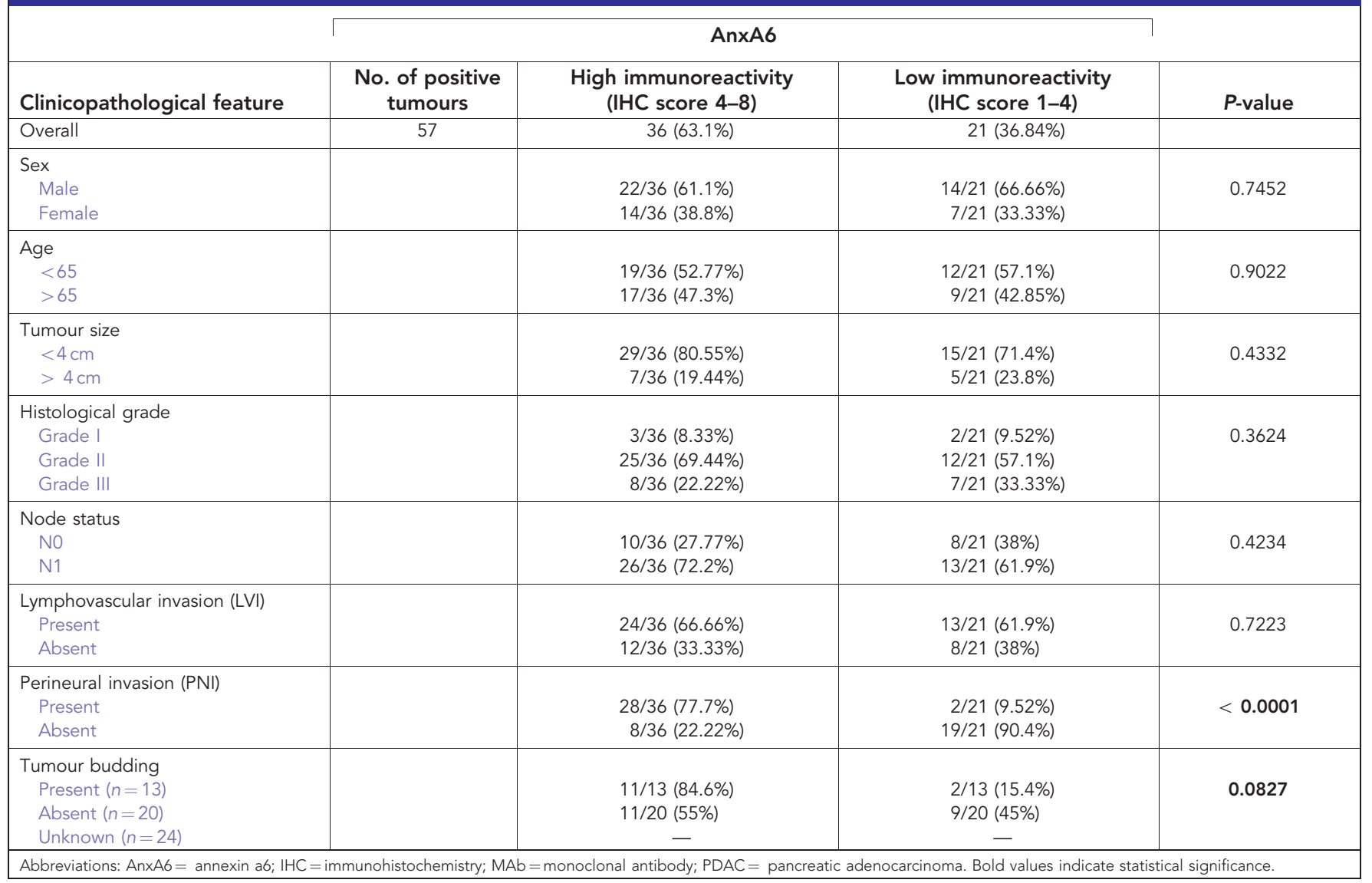

an ADC approach, we are currently assessing if this Mab can induce internalisation of the antibody antigen complex upon binding of AnxA6 at the cell surface, which is critical for any potential ADC targeting approach. We observed AnxA6 immunoreactivity in PDAC tumour stroma in approximately $50 \%$ of tumours. There is emerging evidence that LRP-1 is involved in tumour adhesion and invasion via modulation of ERK and JNK pathways. Although we did not identify LRP-1 (or TSP-1) as an interacting partner of AnxA6 here, it is not unreasonable to speculate that AnxA6 may be mediating effects on invasion through interaction with LRP-1 and subsequent activation of ERK signalling. Interestingly in the context of exploring AnxA6 as a potential antibody target, AnxA6 has been shown to be involved in the regulation of IL-2 signalling and T-cell plasma membrane organisation (Cornely et al, 2016). T cells and immune checkpoints have become the focus of numerous efforts to therapeutically modulate anti-tumour immune response.

To our knowledge, we show for the first time evidence of membrane AnxA6 expression in both colon adenocarcinoma and PDAC (Figure 4B and C), indicating that AnxA6 is specifically and abundantly expressed in both cancers. As we had demonstrated, using MAb 9E1, that targeting AnxA6 can significantly impede PDAC invasion, we hypothesised that AnxA6 may be involved in PDAC tumourgenesis, at least in the early invasive stages of the tumour, we then analysed AnxA6 expression in 57 PDAC tumours. Pancreatic adenocarcinoma (PDAC) is a highly aggressive and chemoresistant tumour and is virtually incurable with current treatment modalities available. The observation of weak AnxA6 expression associated with islet cells in normal pancreas is interesting; $\mathrm{B}$ cells in islets have been demonstrated to express stem cell markers, LGR5 and Nanog both of which are involved in PDAC tumour development (Amsterdam et al, 2013). Strong pERK expression has been reported in islet cells. In early stages of PDAC (Amsterdam et al, 2013), elevated ERK activity, resulting from mutation of KRAS, has been shown to be a common event in PDAC.

AnxA6 was expressed in $100 \%$ of PDAC tumours with intense membrane immunoreactivity observed in 36 out of 57 (63\%) of tumours (Table 2). Higher AnxA6 expression was significantly associated with the presence of PNI $(P<0.0001)$. Perineural invasion (PNI) is a hallmark of PDAC and a strong predictor of poor prognosis. No significant association between high AnxA6 expression and lymph node involvement was revealed despite the highly significant association with PNI. Tumour budding, small groups of individual tumour cells in the stroma near the invasive front of the tumour, is also an indicator of adverse outcome in PDAC. A greater proportion of tumours where tumour budding was present showed high AnxA6 immunoreactivity compared to tumours where tumour budding was absent $(P=0.0827)$ (Figure 4$)$. These results suggested that AnxA6 is likely to be involved at the early stages of PDAC progression (local invasion). Furthermore, PDAC tumours with higher AnxA6 expression showed a clear trend towards a poorer outcome $v s$ those tumours with lower expression of AnxA6 $(P=0.2242)$; however, this failed to reach significance.

In conclusion, our study highlights the use of phenotypic hybridoma screening in the isolation and development of a novel function blocking, inhibitory MAb, 9E1 with anti-cancer activity and identified its target antigen, AnxA6 as a functionally relevant target molecule. Using MAb 9E1, we have shown that AnxA6 shows a tumour restricted tissue expression profile; it is abundantly expressed in a number of aggressive cancers with limited expression across the vast majority of normal tissues. Moreover, we have demonstrated that strong AnxA6 expression is associated with adverse clinicopathological features and outcome in PDAC. The potent anti-invasive effects of MAb 9E1 warrant further 
studies to fully elucidate its mechanism of action in vitro, and suggest that such an antibody targeting approach could be potentially investigated in vivo, in pre-clinical studies. Taken together, our findings suggest that AnxA6 may represent a potential candidate protein for exploration of antibody-mediated targeting for PDAC (and potentially other aggressive cancer types).

\section{ACKNOWLEDGEMENTS}

This work was funded by Enterprise Ireland, project code TD/ 2009/0133; funding is also acknowledged from the St. Lukes' Institute for Cancer Research The authors wish to acknowledge the technical assistance provided by the RCSI Biomedical Facility, Beaumont Hospital, Dublin 9 and Mr. Damian Tiernan, Department of Pathology, RVEEH, Dublin 4.

\section{CONFLICT OF INTEREST}

The authors declare no conflict of interest.

\section{REFERENCES}

Amsterdam A, Raanan C, Schreiber L, Polin N, Givol D (2013) LGR5 and Nanog identify stem cell signature of pancreas beta cells which initiate pancreatic cancer. Biochem Biophys Res Commun 433: 157-162.

Clynes M, Redmond A, Moran E, Gilvarry U (1992) Multiple drug-resistance in variant of a human non-small cell lung carcinoma cell line, DLKP-A. Cytotechnology 10: 75-89.

Cornely R, Pollock AH, Rentero C, Norris SE, Alvarez-Guaita A, Grewal T, Mitchell T, Enrich C, Moss SE, Parton RG, Rossy J, Gaus K (2016) Annexin A6 regulates interleukin-2-mediated T-cell proliferation. Immunol Cell Biol 94: 543-553.

Dudley DT, Li XY, Hu CY, Kleer CG, Willis AL, Weiss SJ (2014) A 3D matrix platform for the rapid generation of therapeutic anti-human carcinoma monoclonal antibodies. Proc Natl Acad Sci USA 111: 14882-14887.

Glynn SA, Gammell P, Heenan M, O'Connor R, Liang Y, Keenan J, Clynes M (2004) A new superinvasive in vitro phenotype induced by selection of human breast carcinoma cells with the chemotherapeutic drugs paclitaxel and doxorubicin. Br J Cancer 91: 1800-1807.

Gonzalez-Munoz AL, Minter RR, Rust SJ (2016) Phenotypic screening: the future of antibody discovery. Drug Discov Today 21: 150-156.

Grewal T, Hoque M, Conway JR, Reverter M, Wahba M, Beevi SS, Timpson P, Enrich C, Rentero C (2017) Annexin A6-A multifunctional scaffold in cell motility. Cell Adh Migr 11: 288-304.

Grewal T, Evans R, Rentero C, Tebar F, Cubells L, de Diego I, Kirchhoff MF, Hughes WE, Heeren J, Rye KA, Rinninger F, Daly RJ, Pol A, Enrich C (2005) Annexin A6 stimulates the membrane recruitment of p120GAP to modulate Ras and Raf-1 activity. Oncogene 24: 5809-5820.

Kinch MS, Kohli M, Goldblatt M, Li WB (2009) Function-first approaches to improve target identification in cancer. Future Oncol 5: 617-623.

Koese M, Rentero C, Kota BP, Hoque M, Cairns R, Wood P, Vila de Muga S, Reverter M, Alvarez-Guaita A, Monastyrskaya K, Hughes WE, Swarbrick A, Tebar F, Daly RJ, Enrich C, Grewal T (2013) Annexin A6 is a scaffold for PKCalpha to promote EGFR inactivation. Oncogene 32: 2858-2872.

Koumangoye RB, Nangami GN, Thompson PD, Agboto VK, Ochieng J, Sakwe AM (2013) Reduced annexin A6 expression promotes the degradation of activated epidermal growth factor receptor and sensitizes invasive breast cancer cells to EGFR-targeted tyrosine kinase inhibitors. Mol Cancer 12: 167-4598-12-167.

Langlois B, Perrot G, Schneider C, Henriet P, Emonard H, Martiny L, Dedieu S (2010) LRP-1 promotes cancer cell invasion by supporting ERK and inhibiting JNK signaling pathways. PLOS ONE 5: e11584.

Larkin A, Moran E, Kennedy SM, Clynes M (2005) Monoclonal antibody 5C3 raised against formalin fixed paraffin-embedded invasive breast tumour tissue: characterisation of its reactive antigen via immunoprecipitation and internal sequencing. J Immunol Methods 303: 53-65.

Leca J, Martinez S, Lac S, Nigri J, Secq V, Rubis M, Bressy C, Serge A, Lavaut MN, Dusetti N, Loncle C, Roques J, Pietrasz D, Bousquet C, Garcia S, Granjeaud S, Ouaissi M, Bachet JB, Brun C, Iovanna JL, Zimmermann P, Vasseur S, Tomasini R (2016) Cancer-associated fibroblast-derived annexin A6 + extracellular vesicles support pancreatic cancer aggressiveness. J Clin Invest 126: 4140-4156.

Luan Z, He Y, Alattar M, Chen Z, He F (2014) Targeting the prohibitin scaffold-CRAF kinase interaction in RAS-ERK-driven pancreatic ductal adenocarcinoma. Mol Cancer 13: 38-4598-13-38.

McBride S, Meleady P, Baird A, Dinsdale D, Clynes M (1998) Human lung carcinoma cell line DLKP contains 3 distinct subpopulations with different growth and attachment properties. Tumour Biol 19: 88-103.

Moulik S, Pal S, Biswas J, Chatterjee A (2014) Role of ERK in modulating MMP 2 and MMP 9 with respect to tumour invasiveness in human cancer cell line MCF-7 and MDA-MB-231. J Tumor 2(2): 87-98.

O'Sullivan D, Henry M, Joyce H, Walsh N, Mc Auley E, Dowling P, Swan N, Moriarty M, Barnham P, Clynes M, Larkin A (2014) 7B7: a novel antibody directed against the Ku70/Ku80 heterodimer blocks invasion in pancreatic and lung cancer cells. Tumour Biol 35: 6983-6997.

Pons M, Grewal T, Rius E, Schnitgerhans T, Jackle S, Enrich C (2001) Evidence for the involvement of annexin 6 in the trafficking between the endocytic compartment and lysosomes. Exp Cell Res 269: 13-22.

Qi H, Liu S, Guo C, Wang J, Greenaway FT, Sun MZ (2015) Role of annexin A6 in cancer. Oncol Lett 10: 1947-1952.

Sakwe AM, Koumangoye R, Guillory B, Ochieng J (2011) Annexin A6 contributes to the invasiveness of breast carcinoma cells by influencing the organization and localization of functional focal adhesions. Exp Cell Res 317: 823-837.

Sandercock AM, Rust S, Guillard S, Sachsenmeier KF, Holoweckyj N, Hay C, Flynn M, Huang Q, Yan K, Herpers B, Price LS, Soden J, Freeth J, Jermutus L, Hollingsworth R, Minter R (2015) Identification of antitumour biologics using primary tumour models, 3-D phenotypic screening and image-based multi-parametric profiling. Mol Cancer 14: 147-0150415-0.

Shevchenko A, Tomas H, Havlis J, Olsen JV, Mann M (2006) In-gel digestion for mass spectrometric characterization of proteins and proteomes. Nat Protoc 1: 2856-2860.

Walsh N, Dowling P, O’Donovan N, Henry M, Meleady P, Clynes M (2008) Aldehyde dehydrogenase $1 \mathrm{~A} 1$ and gelsolin identified as novel invasionmodulating factors in conditioned medium of pancreatic cancer cells. $J$ Proteomics 71: $561-571$.

Wang X, Zhang S, Zhang J, Lam E, Liu X, Sun J, Feng L, Lu H, Yu J, Jin H (2013) Annexin A6 is down-regulated through promoter methylation in gastric cancer. Am J Transl Res. 5: 555-562.

This work is published under the standard license to publish agreement. After 12 months the work will become freely available and the license terms will switch to a Creative Commons AttributionNonCommercial-Share Alike 4.0 Unported License.

Supplementary Information accompanies this paper on British Journal of Cancer website (http://www.nature.com/bjc) 